\title{
Epidemiology, Reported Injury Characteristics of Brain Trauma: Evidences Collected from a Level-One- Trauma Center in Zahedan City, Iran
}

\author{
Minoo Sharbafshaaer ${ }^{1 *(1)}$ \\ ${ }^{1}$ Young Researchers and Elite Club, Zahedan Branch, Islamic Azad University, Zahedan, Iran \\ *Corresponding Author: Minoo Sharbafshaaer, M.Sc., Young Researchers and Elite Club, Zahedan Branch, Islamic \\ Azad University, Zahedan, Iran. Email: minoosharbaf@gmail.com
}

Received September 25, 2020; Accepted February 14, 2021; Online Published March 7, 2021

\begin{abstract}
Background: Traumatic brain injury (TBI) is the damage to brain tissue and disruption of the brain function caused by an external mechanical force as evidenced by documented medical records.

Objectives: The study aimed to estimate the prevalence of TBI through age-groups, severity, and mechanism of TBI.

Methods: This cross-sectional study contained all TBI patients who were admitted at the main level one trauma center hospital located in southeastern Iran.

Results: The number of reported patients was 445 young and old adults with TBI. In total, the mean age of the patients was 32.35 ranging from 17 to $>80$ years. Of the studied patients $361(81.1 \%)$ were male and $84(18.9 \%)$ were women. The most common principal severity of TBI among patients aged 20-29 years was moderate. This corresponds with the age groups that are known to be at higher risk for three levels of TBI. Car accident multiple trauma and head trauma show severity of TBI in both genders.

Conclusion: The research findings determined the lack of ignoring the traffic control system in southeastern Iran that was the main cause of the injury; consequently, the focus of all essentials in traffic management should be considered for this problem. It must be noted that the incidence of TBI is necessary as there remains no cure for mild-to-severe TBI. As the evidence for effectiveness and specific treatment is limited, it must be subjected to demanding research.

Keywords: Traumatic Brain Injuries, Trauma Center, Epidemiology, Injury Characteristics
\end{abstract}

\section{Background}

Traumatic brain injury (TBI) is "the hurt to brain tissue and difficulties with brain function, thus it remains the leading cause of death and disability among trauma patients. Each year, 69 million people in many countries suffer from TBI. ${ }^{1}$ Also, sixty-nine million individuals in the Southeast Asia and Western Pacific regions are undergoing TBI, ${ }^{2}$ while TBI has become the major injury in the military in Iraq and Afghanistan. ${ }^{3}$ Iranian trauma center reported, more than 700000 people were injured in road accidents. The number of traumatic brain injuries by road accidents is almost 20 times higher than the global average. Every 19 minutes, one person dies in a traffic accident in Iran. ${ }^{4}$

Age and gender are known to be strong prognostic factors following TBI, defining age and gender-critical prognostic should be included in TBI research, ${ }^{5}$ as TBI affects these factors. In Iran, the investigations show the distribution of TBI and deaths were more among males rather than females, especially in the elderly age group, thus the males population has been reporting the highest risk of injuries in the young ages. ${ }^{4}$

TBI is acquired from a mechanism of brain injury that precedes the traumatic event, and cause different grades of trauma injuries can be temporary or long-lasting, with central or spread injuries in the brain. Moreover, the severity of TBI based on the Glasgow Coma Scale (GCS) is mainly classified as mild, moderate, or severe grades by the loss of consciousness. ${ }^{6}$ In most epidemiological studies on the severity of TBI, mild traumatic brain injuries have captured a greater degree of severity. ${ }^{7}$ Furthermore, studies declared the more common mechanisms of TBI as car crashes, falls, assaults, motor vehicle, or traffic accidents. ${ }^{8}$ Meanwhile, each year trauma centers in Iran, mention car accidents as the most important cause of head injuries. ${ }^{9}$

\section{Objectives}

The present study tries to investigate the characteristics of brain trauma, also few studies have put effort into to clearing other characteristics of brain trauma specifically for TBI patients in Zahedan, Iran. It is therefore essential to identify characteristics factors (sex, age-group, mechanisms, and severity of TBI) for TBI patients and detect the high-risk factors to improve the quality of services and to establish a trauma care system for patients with brain trauma.

Copyright (C) 2021 The Author(s). This is an open-access article distributed under the terms of the Creative Commons Attribution License (http:// creativecommons.org/licenses/by/4.0), which permits unrestricted use, distribution, and reproduction in any medium, provided the original work is properly cited. 


\section{Methods}

This retrospective study commenced a population-based epidemiologic to study TBI in the capital city of Sistan and Baluchistan province, Zahedan, Iran from March 2017 to October 2017 and reviewed TBI-patients records in Khatam trauma emergency department hospital in Zahedan. The medical records based on the ICD-10-CM items include code S07.1 crushing injury of skull and code S06.9 intracranial injury, TBI-related death was collected based on the death certificate, and demographic data.

GCS was introduced for clinical monitoring following TBI and was subsequently used to grade TBI severity followed by 3-8 severe TBI, 9-12 moderate, and 13-15 mild TBI. ${ }^{10}$

Statistical frequency analyses were performed using SPSS version 25.0. Descriptive Statistics with a 95\% confidence interval (CI) were utilized to estimate the percent and frequency prevalence of age-groups, severity, and mechanism TBI at Zahedan trauma center.

\section{Results}

From a total of 445 hospitalized patients for TBI, the mean age of the patients was 32.35 ranging from 17 to $>80$ years. The patient population included $361(81.1 \%)$ men and 84 (18.9\%) women. In particular, 20-29 years of age was the peak of prevalence in TBI patients (44.5\%) and the young male population with 20-29 years old were reported to be the main high-risk age group number of TBI (Table1). In the severity of TBI, at moderate level of $67.4 \%$ the incidence is higher in patients (Table 2). The distribution rate of the causes of brain injury is very diverse, while the first mechanism of TBI was car-accident-multiple-trauma by $44.0 \%$ and head trauma by 18.9 was the second reason for TBI frequency (Table 3 ).

Furthermore, the most common principal severity of TBI conducted among TBI patients aged 20-29 years old

Table 1. Age Group

\begin{tabular}{lccc}
\hline Group & Variable & Frequency & Percent \\
\hline 1 & $17-19$ & 61 & 13.7 \\
2 & $20-29$ & 198 & 44.5 \\
3 & $30-39$ & 67 & 15.1 \\
4 & $40-49$ & 46 & 10.3 \\
5 & $50-59$ & 28 & 6.3 \\
6 & $60-69$ & 30 & 6.7 \\
7 & $70-79$ & 4 & .9 \\
8 & $\geq 80$ & 6 & 1.3 \\
Total & & 440 & 98.9 \\
\hline
\end{tabular}

Table 2. The Severity of Traumatic Brain Injury

\begin{tabular}{lcc}
\hline & Frequency & Percent \\
\hline Severe & 37 & 8.3 \\
Moderate & 300 & 67.4 \\
Mild & 108 & 24.3 \\
\hline
\end{tabular}

Table 3. Mechanism of Traumatic Brain Injury

\begin{tabular}{lcc}
\hline & Frequency & Percent \\
\hline Head-trauma & 84 & 18.9 \\
Car-accident-multiple-trauma & 196 & 44.0 \\
Car-accident, head-trauma & 16 & 3.6 \\
Car-accident, head-trauma, MI & 1 & .2 \\
Car-accident-multiple-trauma, IFI & 1 & .2 \\
Car-accident-multiple-trauma, head-trauma & 2 & .4 \\
Motor-accident, head-trauma & 11 & 2.5 \\
ICH & 1 & .2 \\
Motor-accident, MI & 19 & 4.3 \\
Motor-accident & 7 & 1.6 \\
Car-accident & 50 & 11.2 \\
\hline Multiple-trauma & 13 & 2.9 \\
Motor-accident, multiple-trauma & 14 & 3.1 \\
\hline Falling & 30 & 6.7 \\
\hline Total & 445 & 100 \\
\hline CH-invasve & & \\
\hline
\end{tabular}

$\mathrm{ICH}=$ invasive fungal infection; $\mathrm{IFI}$, invasive fungal infections; $\mathrm{MI}$, monitoring intracranial

was at the moderate level, also the males and females' population with this age group have been known to be at high risk for three degrees of TBI (Figure 1). Most of the traumatic brain injuries that took place by car accident multiple trauma and head trauma show the severity of TBI in both genders (Figure 2).

\section{Discussion}

The present study estimates the number of TBI patients at the main level-one trauma center hospital located in Zahedan, southeastern Iran. The males continue to have higher rates of TBI compared with females. Whereas, integrating sex and age groups in research is recognized as leading to better science, and ultimately better clinical practice. Other research confirmed that sex, age group, severities, and mechanisms of TBI could be the most important factors for medical care. ${ }^{11}$

Therefore, sex, age groups, severities, and mechanisms of the TBI analytical approach are important to understand TBI so as to improve the quality of life outcomes and rehabilitation for TBI survivors. ${ }^{12}$ Males had a higher rate of TBI incidence than females. Also, similar research studies argued that sex and age group are the most commonly identified risk factors, regarding sex and age group relationship, males are nearly three times more likely to suffer a TBI than females. ${ }^{13}$ Generally, the males reported more TBI injures. ${ }^{14}$ Also, at Zahedan trauma center 20-29 age group experienced TBI injuries more than other age groups, TBI evidence in Brazil indicates the highest effective epidemiologic vigilance of TBI was in young adults aged 20-29. ${ }^{15}$

This study had a high proportion of patients with a moderate degree of TBI; meanwhile, in Norway trauma centers, moderate TBI was reported as a major degree of head injuries where they recorded history of staying in an 


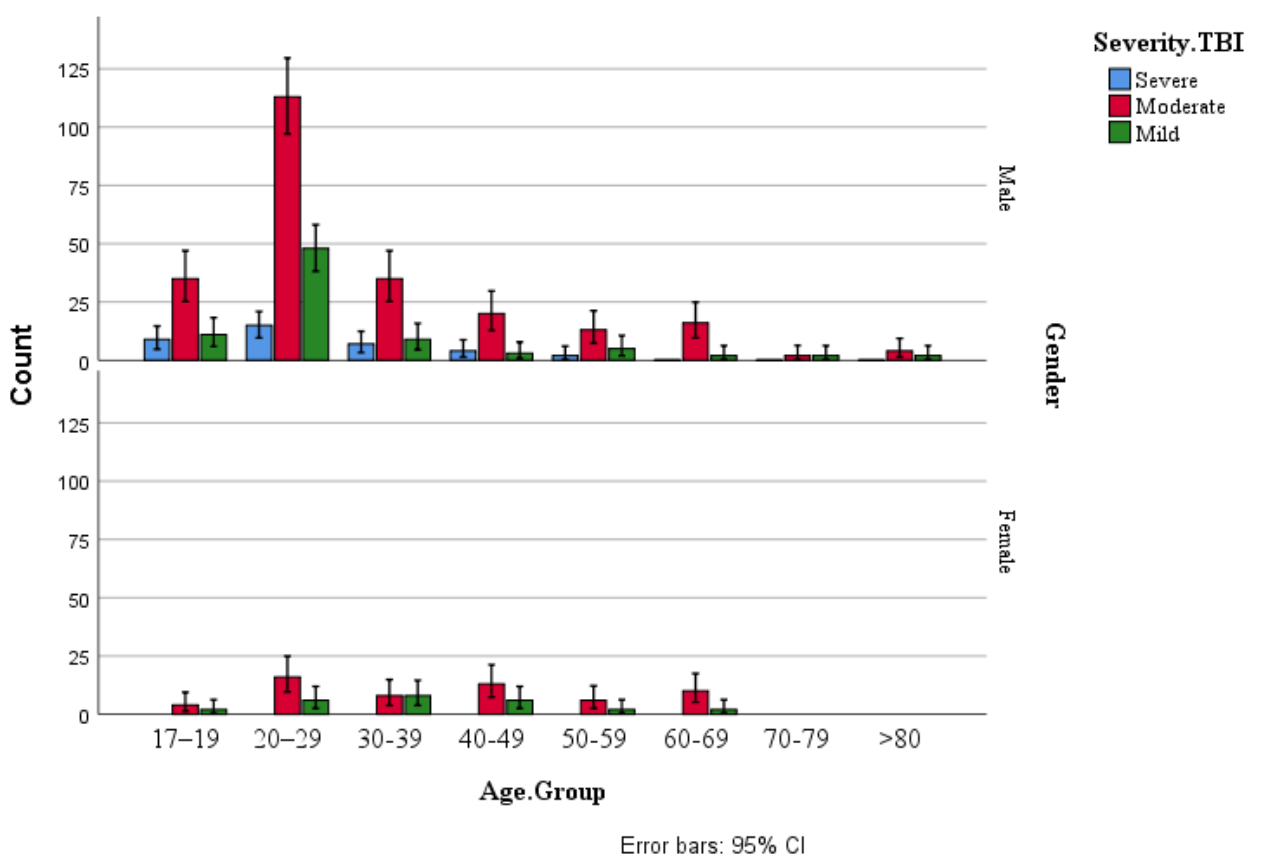

Figure 1. Severity of TBI and Age-group by Gender.

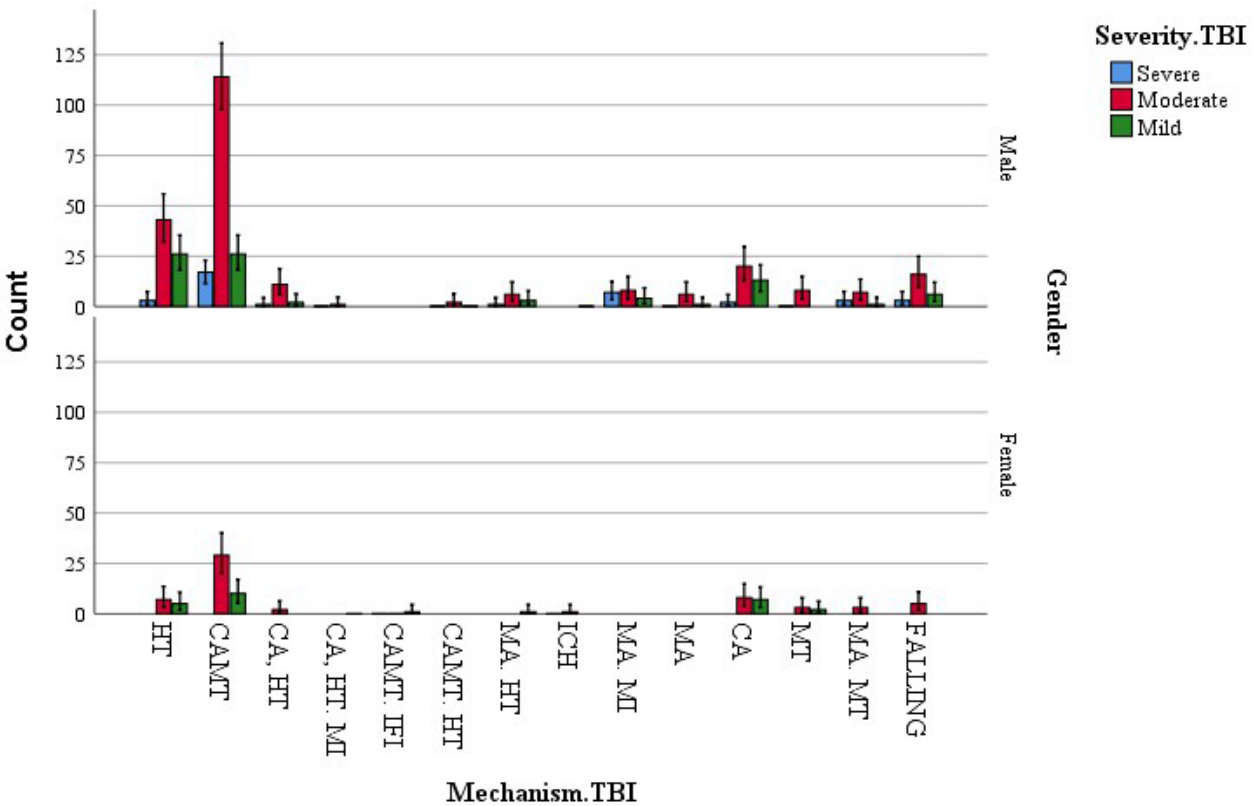

Error bars: $95 \% \mathrm{Cl}$

Figure 2. Severity of TBI and Brain Trauma Mechanism by Gender.

ICU with differentiated hospital care. ${ }^{16}$

The result showed the main mechanisms of TBI were vehicle crashes and head trauma. Additionally, in Arizona, traffic vehicle crashes are the most common type of injury in emergency room visits, hospitalizations, and deaths related to TBI. ${ }^{17}$ Furthermore, in the Netherlands more than half of the TBI patients were involved in road traffic crashes. ${ }^{18}$

The insights into the causes, patterns, and distributions of TBI patients from this study will be extremely helpful in policymaking, research, health management, and rehabilitation at the national level in our country and other developing nations. These methods provide promises for future studies to further clarify the true epidemiology of characteristics injury of brain trauma, which in turn will guide the development of clinical endpoints for diagnostic and the outcome of studies and data collection are of great importance in TBI studies, and is crucial to advance the care for TBI patients in the future. Current approaches are often crude and widely divergent, using only momentary or summary measures. Here, we see a great need for the use of advanced information technology, and further research into the best approaches to these analyses. Further work is needed to determine the best measure of outcome 


\section{Research Highlights}

What Is Already Known?

Traumatic brain injury (TBI) is a field of medicine with one of the greatest unmet needs. Globally, its incidence is increasing, mainly due to increasing traffic in lowand middle-income countries.

\section{What Does This Study Add?}

The traffic control system in Sistan and Baluchistan province was the main cause of the injury; consequently, the focus of all essentials in traffic management should be considered for traumatic brain injuries troubles, also it would be the most important steps forward in the field of clinical care of TBI.

predictor of true mortality rates and definable outcomes in more endangered TBI group people.

\section{Conclusion}

The research findings determined the lack of ignoring the traffic control system in southeastern Iran that was the main cause of injury; consequently, the focus of all essentials in the traffic management should be considered for this problem. It must be recollected that the incidence of TBI is necessary as there remains no cure for mild-to-severe TBI. Since the evidence for effectiveness and specific treatment is limited, it must be subjected to demanding research.

\section{Authors' Contributions}

Study design: MSI, AN; Data collection: YN; Data analysis: YN; Manuscript writing: ED; Critical revisions for important intellectual content: ED.

\section{Conflict of Interest Disclosures}

The authors declare that they have no conflicts of interest.

\section{Ethical Approval}

The study protocol was approved by the Zahedan University of Medical Sciences and the statement of Head's Khatam Hospital. Collecting data was performed by considering patients' history from emergency hospital admission.

\section{Acknowledgements}

We are grateful to the staff of Khatam hospital for their kind cooperation in this study.

\section{References}

1. Sherer M. Traumatic brain injury. In: Budd M, Hough S, Wegener S, Stiers W, eds. Practical Psychology in Medical Rehabilitation. Cham: Springer; 2017. p. 103-108. doi:10.1007/978-3-31934034-0_12.

2. Azim A, Joseph B. Traumatic brain injury. In: Salim A., Brown C., Inaba K., Martin M, eds. Surgical Critical Care Therapy. Cham: Springer; 2018. p. 1-10. doi:10.1007/978-3-319-
71712-8_1.

3. Dewan MC, Rattani A, Gupta S, et al. Estimating the global incidence of traumatic brain injury. J Neurosurg. 2018:1-18. doi:10.3171/2017.10.jns17352.

4. Ansari M, Bayan L, Gorji A. The impact of road accidents on brain injury. The Neuroscience Journal of Shefaye Khatam. 2016;4(3):103-110. doi:10.18869/acadpub.shefa.4.3.103. [Persian].

5. Dhandapani S, Manju D, Sharma B, Mahapatra A. Prognostic significance of age in traumatic brain injury. J Neurosci Rural Pract. 2012;3(2):131-135. doi:10.4103/0976-3147.98208.

6. McKee AC, Daneshvar DH. The neuropathology of traumatic brain injury. Handb Clin Neurol. 2015;127:45-66. doi:10.1016/ b978-0-444-52892-6.00004-0.

7. Leo P, McCrea M. Epidemiology. In: Laskowitz D, Grant G, eds Translational Research in Traumatic Brain Injury. Boca Raton, FL: CRC Press/Taylor and Francis Group; 2016.

8. Mistry J, Murray N. Traumatic brain injury in adults. InnovAiT. 2017;10(10):608-613. doi:10.1177/1755738017710043.

9. Azami-Aghdash S, Sadeghi-Bazargani $H$, Shabaninejad $H$, Abolghasem Gorji H. Injury epidemiology in Iran: a systematic review. J Inj Violence Res. 2017;9(1):27-40. doi:10.5249/jivr. v9i1.852.

10. Basauhra Singh HK, Chong MC, Thambinayagam HC, et al. Assessing nurses knowledge of Glasgow Coma Scale in emergency and outpatient department. Nurs Res Pract. 2016;2016:8056350. doi:10.1155/2016/8056350.

11. Sharbafshaaer $M$, Bhattacharyya $S$. Neurocognitive dysfunction in head injured patients, does it reveal various outcomes in both sexes and age-groups? Int Clin Neurosci J. 2018;5(4):121 125.

12. Colantonio A. Sex, gender, and traumatic brain injury: a commentary. Arch Phys Med Rehabil. 2016;97(2 Suppl):S1-4. doi:10.1016/j.apmr.2015.12.002.

13. Gardner AJ, Zafonte R. Neuroepidemiology of traumatic brain injury. Handb Clin Neurol. 2016;138:207-223. doi:10.1016/ b978-0-12-802973-2.00012-4.

14. Frost RB, Farrer TJ, Primosch $M$, Hedges DW. Prevalence of traumatic brain injury in the general adult population: a meta-analysis. Neuroepidemiology. 2013;40(3):154-159. doi:10.1159/000343275.

15. de Almeida CE, de Sousa Filho JL, Dourado JC, Gontijo PA, Dellaretti MA, Costa BS. Traumatic brain injury epidemiology in Brazil. World Neurosurg. 2016;87:540-547. doi:10.1016/j. wneu.2015.10.020.

16. Lund SB, Gjeilo KH, Moen KG, Schirmer-Mikalsen K, Skandsen $\mathrm{T}, \mathrm{Vik}$ A. Moderate traumatic brain injury, acute phase course and deviations in physiological variables: an observational study. Scand J Trauma Resusc Emerg Med. 2016;24:77. doi:10.1186/s13049-016-0269-5.

17. University of Arizona College of Engineering. Which Car Crashes Cause Traumatic Brain Injury? New Tool to Calculate Likelihood of Traumatic Brain Injury After a Vehicle Collision. ScienceDaily; 2019. https://www.sciencedaily.com/ releases/2019/04/190424172645.htm.

18. Bossers SM, Boer C, Bloemers FW, et al. Epidemiology, prehospital characteristics and outcomes of severe traumatic brain injury in the Netherlands: the BRAIN-PROTECT study. Prehosp Emerg Care. 2020:1-12. doi:10.1080/10903127.202 0.1824049 . 